\title{
Making the future
}

\author{
Welcome to the first issue of Nature Synthesis; a home for new and important syntheses of molecules and materials \\ that can make the world a better place.
}

$\mathrm{C}$ hemists and materials scientists often start their research journeys with the making of things. Typically, their aim is to either delve into the unknowns of a substance's chemical and physical properties to further our fundamental understanding, or to make a product that could ultimately benefit society. In some cases, it's the final product that's the most important part of the work, but in others it's the process of reaching it - the synthesis - where the main advance lies. Nature Synthesis will publish articles that fit into either of these categories, as well as those cases where both the target compound and the journey to it are exceptional.

But what factors make a synthesis important and/or interesting? In terms of practical considerations, it could be that it is low cost, energy- or time-efficient, easily scalable, operationally straightforward or safer than related methods. From a fundamental scientific perspective, it could be a synthesis that allows access to new classes of molecules and materials or enables products to be made with greater selectivity than other approaches. It is also crucial to acknowledge that different communities will value different things; what makes a synthesis attractive to a researcher in industry may not be the same as what piques the interest of those in academia.

Synthesis is a common pursuit across different scales and substances; the same reactions that can be used to make small molecules can also be applied to polymeric systems and extended framework materials. One such example is the concept (and class of transformations) known as click chemistry, the impact of which on both molecular and materials science is discussed in a Comment in this issue. Additionally, an example of synthetic innovation in materials science is described in an Article in this issue, where covalent-organic frameworks
(COFs) are prepared using a sonochemical approach rather than typical solvothermal methods. Not only does the use of sound waves enable known COFs to be prepared in shorter times and at lower temperatures than reported previously, but it also leads to improved crystallinity and porosity, which translates into better catalytic performance. Moreover, the generality of this synthetic approach is demonstrated by showing that it can be used to make new COFs that have not been reported before.

\section{"Synthesis is entering a \\ new age and, in the future, algorithms will help guide us through chemical space."}

Arguably, launching a Nature Research journal on the topic of synthesis is long overdue. Chemical synthesis as a discipline has evolved significantly over the past century and materials synthesis has undergone a huge amount of growth since the emergence of nanotechnology in the last few decades. However, with the computational power now available to researchers, synthesis is entering a new age and, in the future, algorithms will help guide us through chemical space. With this in mind, we think Nature Synthesis is arriving on the scene at an opportune moment; coinciding with a time when machine learning and automation are increasing the pace of discovery in the field. On the topic of machine learning, an Article in this issue reports a computer algorithm to identify potential new sequences of iterative reactions and then a handful of them are experimentally validated. Since iterative reaction sequences lend themselves to automation, the discoveries reported here have the potential to be applied more broadly in this context.
As well as describing the creation of molecules and materials, Nature Synthesis will also focus on the degradation, recovery and recyclability of these products. Some natural resources are finite and others have significant environmental or societal costs related to their extraction or preparation. As a result, there are important considerations about the treatment of materials to ensure a sustainable future. This theme is introduced in a World View, with a focus on the importance of materials design, the infrastructure in which materials are used, and the environment in which they are disposed of, in a necessary effort to achieve sustainable materials recovery.

Whatever your career stage or role in current research, all of our ways of working have changed considerably since the start of the global pandemic. Our ability to interact has been altered and priorities have shifted - for some, this has led to projects to fight COVID-19. In a News Feature, the factors and events around the repurposing of drugs for tackling COVID-19 are discussed.

As we close our first issue, the 2021 Nobel Prize in Chemistry has been awarded to Benjamin List and David MacMillan for the development of asymmetric organocatalysis. On the announcement of the prize, Johan Åqvist, the chair of the Nobel Committee for Chemistry said, "This concept for catalysis is as simple as it is ingenious, and the fact is that many people have wondered why we didn't think of it earlier." Going forward, we hope that ingenious work described in Nature Synthesis will be adopted by, and prove useful to, those in the synthetic community, as well as inspire the creation of new methods, molecules and materials for the benefit of humankind.

Published online: 12 January 2022 https://doi.org/10.1038/s44160-021-00022-z 